\title{
Self-Perceived Performance-Based Training Needs of Senior Nurse Managers Working in United Christian Hospital: A Cross-Sectional Exploratory Study
}

\author{
Lap Fung Tsang ${ }^{1 *}$, So Yuen Alice Sham ${ }^{1}$, Sheung Lan Winnie Law ${ }^{1}$, Ching Yi Sze ${ }^{1}$, Siu Keung Tang ${ }^{2}$, \\ Ching Yan Kong ${ }^{3}$, Shuk Chun Irene Ho ${ }^{1}$, Suk Ying Peggy Wong ${ }^{4}$ and A Marie Tarrant ${ }^{5}$
}

${ }^{1}$ Nursing Services Division, United Christian Hospital, China

${ }^{2}$ Department of Medicine \& Geriatrics, United Christian Hospital, China

${ }^{3}$ Orthopaedics \& Traumatology Department, United Christian Hospital, China

${ }^{4}$ Nursing Services Department, Hong Kong Special Administrative Region, China

${ }^{5}$ School of Nursing, The University of Hong Kong, China

*Corresponding author: Lap Fung TSANG, PDANP (M\&L), MMMR, MN, BSN, RN, Nursing Services Division, Rm. 4B, 4/F, Block L, United Christian Hospital, 130 Hip Wo Street, Kwun Tong, KLN, Hong Kong Special Administrative Region, China, Tel: 852-52153340, Fax: 852-39495540, E-mail: tsanlf1@ha.org.hk

\begin{abstract}
An increasing demand for quality of clinical service and safety of clinical procedures in hospitals has become a burden to healthcare staff. Training resources is sometimes wrongly allocated and are inadequate to meet the nurses' actual needs. No study was conducted to explore self-perceived training needs from senior level managers towards three domains: personal growth, professional development and services development. A cross-sectional survey study was conducted from $3^{\text {rd }}$ to $23^{\text {rd }}$ August 2015 in Hong Kong to explore self-perceived training needs from 62 senior nurse managers towards three domains: personal growth, professional development and services development. The findings suggested that senior level managers need a variety of trainings to achieve a high performed leader. The managerial performance characteristics was correlated to its training needs $(r=-0.39, p=0.002)$ and the professional performance characteristics was correlated to its training needs $(r=-0.38, p=0.003)$. Nursing professional development specialist can base on a structural training framework to meet their needs in the upcoming 3 years.
\end{abstract}

\section{Keywords}

Training needs, Senior nurse managers, Personal growth, Professional development, Service development

\section{Introduction}

An increasing demand for quality of clinical service and safety of clinical procedures in hospitals has become a burden to healthcare staff. Senior management is responsible to work out strategies for improving the personal competency and work performance of personnel. Being the greatest asset of an organization, staff is critical to the continual health of the organization, in which nurses are the biggest party in the workforce [1]. In order to develop nurses to reach the achievable competency at different levels, Nursing Services Division (NSD), thus, emphasizes on staff education and their professional development.

However, the resources allocated specifically for education are always not adequate to meet the nurses' actual needs. Senior management therefore needs to prioritize the areas that are worthwhile to invest the limited training funding on. It is crucial that education is tailored to meet the specialized needs of the organization and the individual trainees [2]. Nevertheless it is rare to have a report about the need of education from the perspectives of senior level manager in the local context. 
Training Needs Analysis (TNA) survey is commonly used to identify training needs. It is an effective method to determine who and what should be trained based on the perspective of users when planning in training and development [3]. The use of a TNA exercise can support the development of a fit-for-purpose programme of information skills training [4]. Although it was conducted to explore the overall training and educational needs of the nursing staff, it was based on the perspective of the senior management rather than the perceived needs from the nurses [5]. If organizations develop and organize training without recognizing a needs assessment, it may result in over training, under training or just missing the point all together [1]. Therefore, the TNA is a primary step to provide important information in the development of education programme in order to meet the demands of nurses [6]. Hence, the information can facilitate the design of a structural framework that guides senior management to organize appropriate education programmes.

\section{Background}

Hospital Authority (HA) has constructed a performance characteristic for senior level managers, such as Department Operation Manager (DOM), Nurse Consultant (NC), Senior Nurse Officer (SNO), and Ward Manager (WM) to review their performance development. A set of performance characteristics comprised of general managerial and professional performance characteristics are specific to assess the performance of the senior level managers [7].
From December 2013 to March 2014, a total of three focus group interviews composed of general manager (nursing), senior nurse officer, nurse consultant, department operation manager and advanced practice nurse were conducted. The research team chose this method because it enabled access to a number of nurses, and all data and ideas gathered could be explored, confirmed, reinforced or contradicted within the group at the same time $[8,9]$. A group size ranged from 8 to 12 nurses was chosen to ensure that group members had an opportunity for valuable input $[8,9]$. Trigger and open-end questions for the focus group interviews were formulated based on development directions of the Hospital Authority (HA) and United Christian Hospital (UCH), professional practice perceived from members on different levels of nurses, and issues raised in relevant literatures and refined by the research team. Each group had the same researcher and the third author who transposed the conversation in real time into a word document. Group dynamics were discussed and consensuses made by the second author and field notes were written immediately after each group. A document was sent out to the group members to validate the content of the conversations and the directions of trainings to be focused. Finally, three domains including professional development, personal growth and clinical services development were identified and incorporated into the performance characteristics framework developed by HA and training needs based on the existing available training programmes in $\mathrm{UCH}$ to develop a questionnaire for the study (Figure 1).

\section{Framework of the PD\&T in UCH}
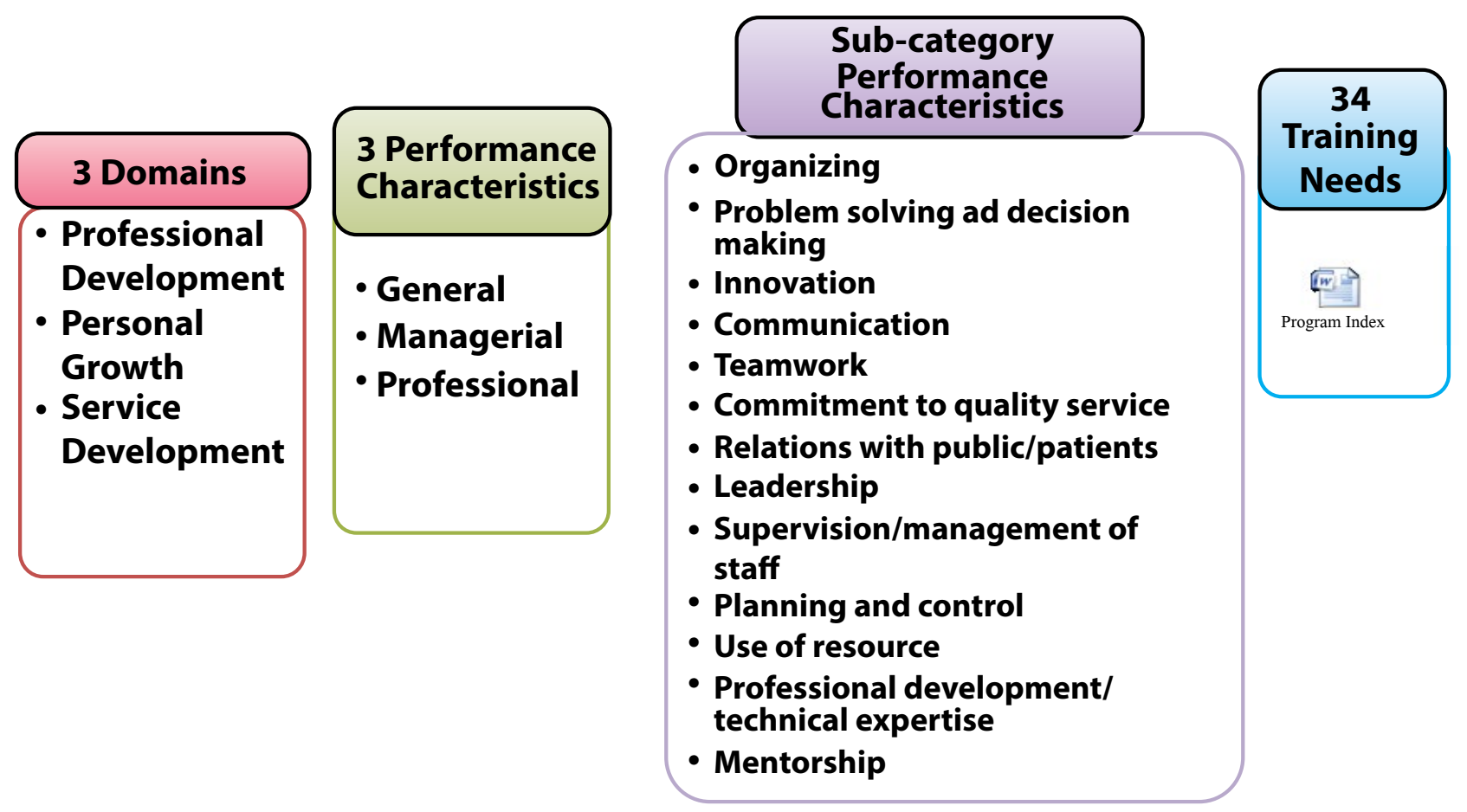

Figure 1: Design of the questionnaire for the study. 
A professional development is referred to a constant commitment to maintain specific skill levels and career paths, and to ensure that a nurse's skills and knowledge are updated and relevant [10]. It is essential for a nurse to commit to professional development for delivering safe and effective health care [11]. Moreover, opportunities of professional development offered by organization for nurses may increase nurse retention [12]. In addition, it is challenging to invest certain resources to develop nursing professions. An effectiveness of professional development is dependent on self-motivating and valued by nurses [13]. As a result, the TNA is a useful way to determine the training that is required based on nurses' lack of necessary knowledge, skills and attitude towards their job [14].

Personal growth is defined as a journey by which people change and develop throughout the life span [15]. During the growth process, it can involve various parts of self, such as cognitions, behaviors and/or emotions [16]. Nurses perceived these changes from work, family and whenever possible affects their capabilities to perform well. The performance characteristics constructed by $\mathrm{HA}$ as mentioned above did not address personal growth. On the other hand, as it is deemed necessary to master new athletic skills, and to manage stressors and challenges encountered throughout life [16], the related to trainings are incorporated into the domain of personal growth, one of the three identified performance characteristics.

A clinical services plan illustrates a cluster's key strategies and directions for the provision of high quality and efficient services in meeting the long term healthcare needs of community. The commitment and wish of healthcare providers for enhancements in the services for their patients are epitomized by the new service models. Different trainings and supports to this kind of service development are necessary to enhance nursing skills in taking care of patients. Therefore, clinical services development serves as a guide for the making of training framework that affects future training needs and prioritization.

\section{Aims of this Research}

The aim of this study was to explore the performance characteristics training needs of senior level managers working in United Christian Hospital (UCH), a district public HA hospital in Hong Kong. The main objectives of this study were: (1) To explore the self-perceived performance characteristics of senior level managers; (2) To explore the self-perceived training needs on required performance characteristics including general, managerial, and professional performance from the senior level managers; (3) To understand the variation of training needs perceived from DOM/ NC/ SNO and WM, and (4) To formulate a performance characteristics professional development and training framework in $\mathrm{UCH}$.

\section{Methods}

\section{Research design}

This is a cross-sectional exploratory study from 73 senior level managers including DOM, NC, SNO and WM, who were invited to complete a self-administered questionnaire from $3^{\text {rd }}$ to $23^{\text {rd }}$ August 2015 in $\mathrm{UCH}$.

\section{Participants}

A total of 73 senior level managers were invited to take part in the study. Nurses who were part-time employment, and on leave including maternity leave, annual leave, study leave or no payment leave during the period of data collection were excluded. A 20-minute briefing session about the design of the study and instruction to fill in the questionnaire was given by the first author and third co-author prior to the period of data collection.

\section{Ethical considerations}

The study was approved by the Research Ethics Committee (Kowloon Central/Kowloon East) (KC/KE-150050/ER-2). Consent was implied by completion of the questionnaire. All participants were voluntary and had every right to participate or refuse without any reason. Anonymity was preserved for questionnaire distribution and collection. The protocol complied with the International Conference on Harmonisation - Guideline for Good Clinical Practice. To protect the privacy of respondents, all study data were handled in line with HA/Hospital's policy in handling/storage/destruction. Electronic data were saved in secured computer of the hospital with restricted access. All the raw data will be destroyed after 3 years of the completion of the study.

\section{Procedures}

The focus of the study was to explore the performance characteristics and training needs from senior level managers directly. Therefore, the questionnaire was composed of three parts including demographic data, self-perceived performance characteristics, and self-perceived training needs. An additional part of training needs from the perspectives of DOM/SNC/SNO for WM was attached in the questionnaire.

Demographic data included gender, age, academic qualification, working unit, number of years in present unit, number of years in current rank and number of years in $\mathrm{UCH}$. Three sub-performance characteristics categories including general, managerial, and professional performance for a total of 20 items were focused. The participants needed to rate from 1 (significant improvement in performance is essential to meet the required standard for the job) to 4 (frequently exceeds the required standard for the job).

Self-designed questionnaires of perceived training needs were employed for DOM, NC, SNO and WM with 
a 34-item instrument. Based on the trainings organized by NSD and HA, these needs were framed against three major domains including professional development, personal growth, and service development. The participants identified the training needs using a 10-point Likert Scale (from 1 indicated "No Need" to 10 indicated "Greatest Need") and they were encouraged to make suggestions about the training needs according to their ranks and years of experience.

Fifty-six items of training need rated by senior nurse managers on $\mathrm{EN}$ and $\mathrm{RN}$ were expressed in a dichotomous (yes/no) response format. Sixty items of training need rated by senior level managers on APN, NO, and NS were expressed in a dichotomous response format, of which were categorized into less than 5 years of experience and equal to or more than 5 years of experience.

\section{Data analysis}

Statistical analysis was performed using the SPSS 23.0 statistical software for Windows (IBM Corp. Released, Armonk, NY, USA, 2015) [17]. The data files were checked for discordant values and contraindications by the first author and a statistician not involved in the study. Descriptive data analysis for nominal variables and ordinal variables were expressed in frequencies and per cents whereas continuous variables were expressed in means and standard deviations. The t-test was used for evaluating the difference of self-perceived performance and training needs in terms of year of experience with less than 5 from equal to or more than 5 . Correlation test was used to determine the consistence between self-perceived performance and training needs.
The level of significance was set at $P<0.05$. A sample size of 70 was estimated using a confidence level at $95 \%$ in a confidence interval of $5.0 \%$ in an exact population size of 86 DOMs, NCs, SNOs and WMs [18].

\section{Validity and reliability}

It is indispensable to measure and report the content validity of questionnaires because of a creation of confidence in measurements of the variables of interest [19]. The questionnaires had undertaken a content validity by three senior nurses to ensure a satisfactory level of content validity scale, rating from 1 (irrelevant), 2 (somewhat relevant), 3 (relevant), to 4 (very relevant) $[20,21]$. The degree of relevance among the domains, the competencies and the training needs in the questionnaire was indicated by a former principal of Institute of Advanced Nursing School, a Chief Nursing Officer (Nursing Education) and MT. The three senior nurses and another seven nurses who worked in NSD as an Executive Partnership (EP) were invited to conduct face validity so that the questionnaire appeared to be appropriate to the study purpose and content [21]. Mean relevance scores of each training need was calculated. It was compromised amongst researchers that the mean score of a training need should be at least 3.0 to justify inclusion into the questionnaire. CVI was calculated for each item under various competencies and for the overall questionnaire. Regarding conciseness, design, information, content and understandability of the two questionnaires, the results from the expert panel yielded an overall CVI of 3.8. Test-retest reliability was estimated by completing the same tool by the first author and the

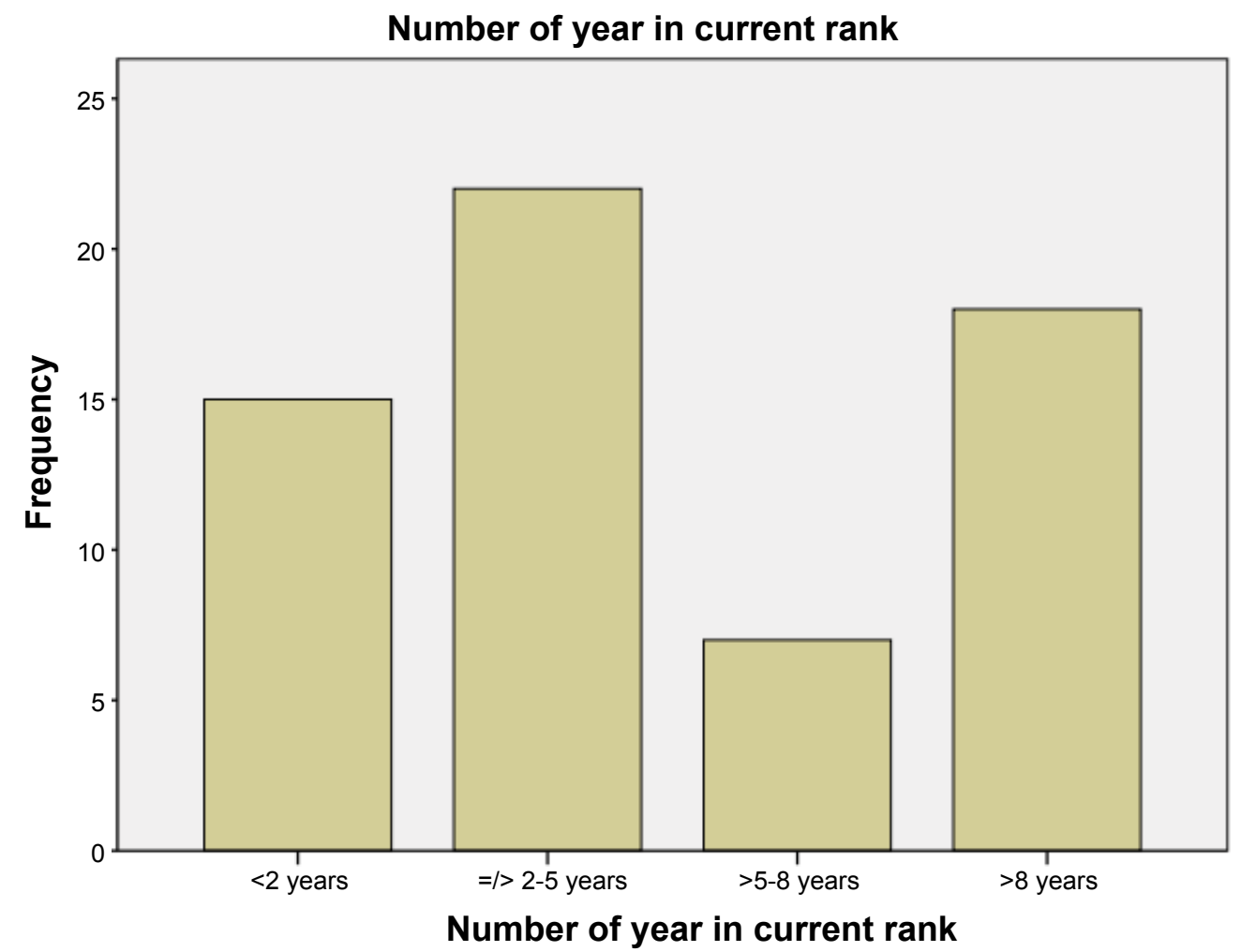

Figure 2: The working experience level in current rank of DOM/NC/SNO/WM. 
forth co-author in an interval of one week. A Cronbach's alpha of more than 0.70 was considered good consistency [22]. The Cronbach's alpha for the general, managerial, and professional performance characteristics were $0.96,0.93$, and 0.94 respectively.

\section{Results}

The response rate from DOM/NC/SNO/WM of the study was $84.9 \%$. All questionnaires from the above ranks were valid for the purposes of the study. The distribution of working experience in current rank of DOM/ NC/SNO/WM was shown in the Figure 2.

Mean of managerial performance characteristics was the lowest amongst other performance characteristics so as to acquire the highest training needs on this area (Table 1). A correlation test between self-perceived performance characteristics and training needs was performed. The managerial performance characteristics was correlated to the training needs under this characteristics $(r=-0.39, p=0.002)$ and the professional performance characteristics was correlated to the training needs under this characteristics $(r=-0.38, p=0.003)$.

\section{Self-perceived performance characteristics}

The overall mean of self-perceived performance characteristics was 3.03. The means of general, managerial, and professional performance characteristics were $3.06,2.87$ and 3.14 respectively. The performance items including organizing, problem solving and decision making, innovation, and communication (verbal and written) under general performance characteristics were necessary to be improved in order to meet the required standard for the job when comparing to the overall mean of self-perceived performance characteristics. Foresight, leadership, supervision/management of staff, planning and control, and use of resources under managerial performance characteristics as well as judgment under professional performance characteristics was necessary to be improved when comparing to the overall mean of self-perceived performance characteristics (Figure 3, Figure 4 and Figure 5).

\section{Self-perceived training needs}

The overall mean of self-perceived training needs was 6.5. The means of training needs under general, managerial, and professional performance characteristics were 6.6, 6.77 and 6.14 respectively. Senior level managers reflected that they needed more training on the areas such as media management, creating and leading a culture of innovation, overseas study visit, presentation skills to public/patients,

Table 1: Comparing self-perceived performance characteristics and self-perceived training needs with the number of year in present rank.

\begin{tabular}{|l|l|l|l|l|l|l|}
\hline \multirow{2}{*}{ Performance characteristics } & \multicolumn{3}{l}{ </=5 years } & \multicolumn{3}{l|}{ Statistical analysis } \\
\cline { 2 - 7 } & mean & SD & mean & SD & t-test & p-value \\
\hline General & 3.03 & 0.4 & 3.09 & 0.46 & 0.54 & $>0.05$ \\
\hline Managerial & 2.77 & 0.53 & 3.01 & 0.54 & 1.70 & 0.1 \\
\hline Professional & 3.10 & 0.44 & 3.21 & 0.53 & 0.87 & $>0.05$ \\
\hline Training needs under general & 6.74 & 1.49 & 6.27 & 1.77 & 1.10 & $>0.05$ \\
\hline Training needs under managerial & 6.97 & 1.69 & 6.46 & 1.84 & 1.10 & $>0.05$ \\
\hline Training needs under professional & 6.16 & 2.0 & 6.10 & 1.80 & 0.13 & $>0.05$ \\
\hline
\end{tabular}

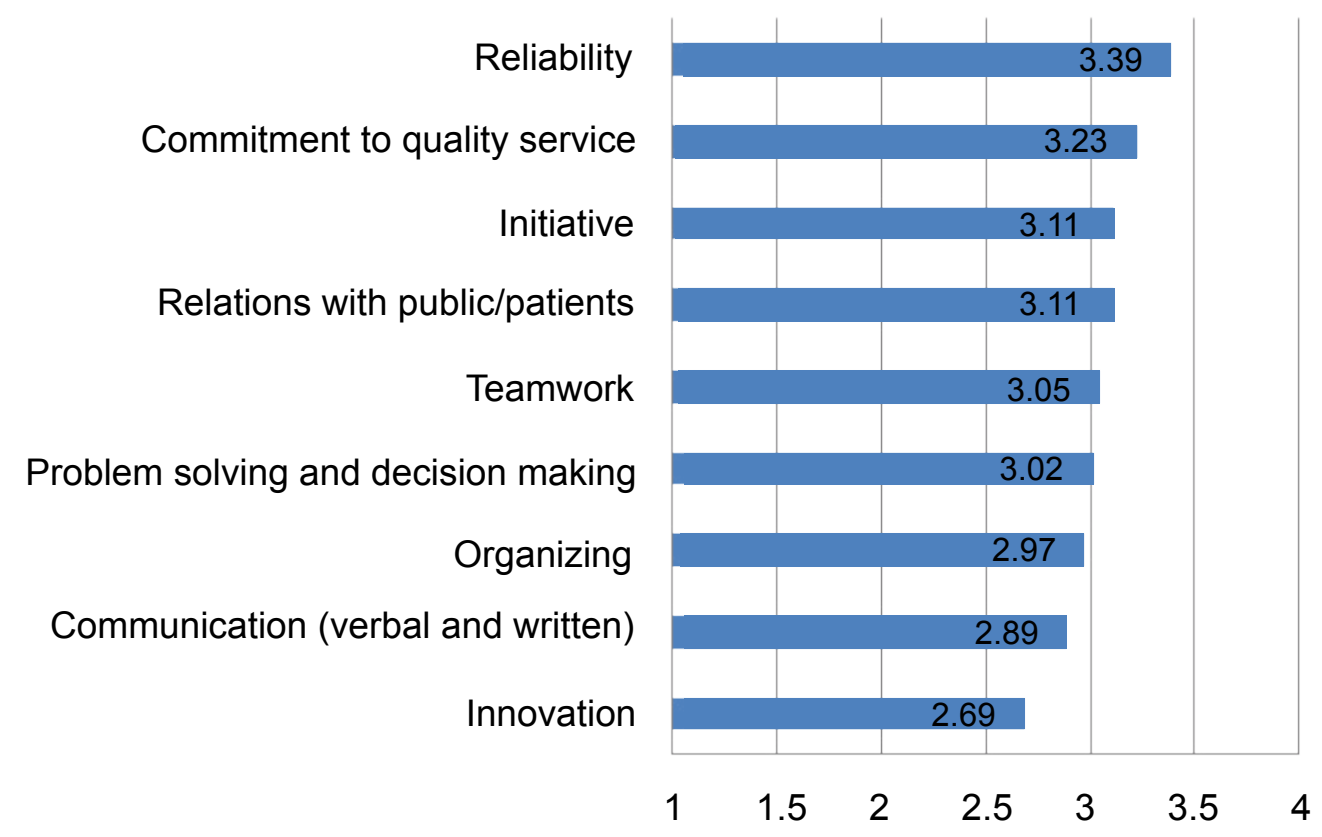

Figure 3: Mean of performance under general performance characteristics. 


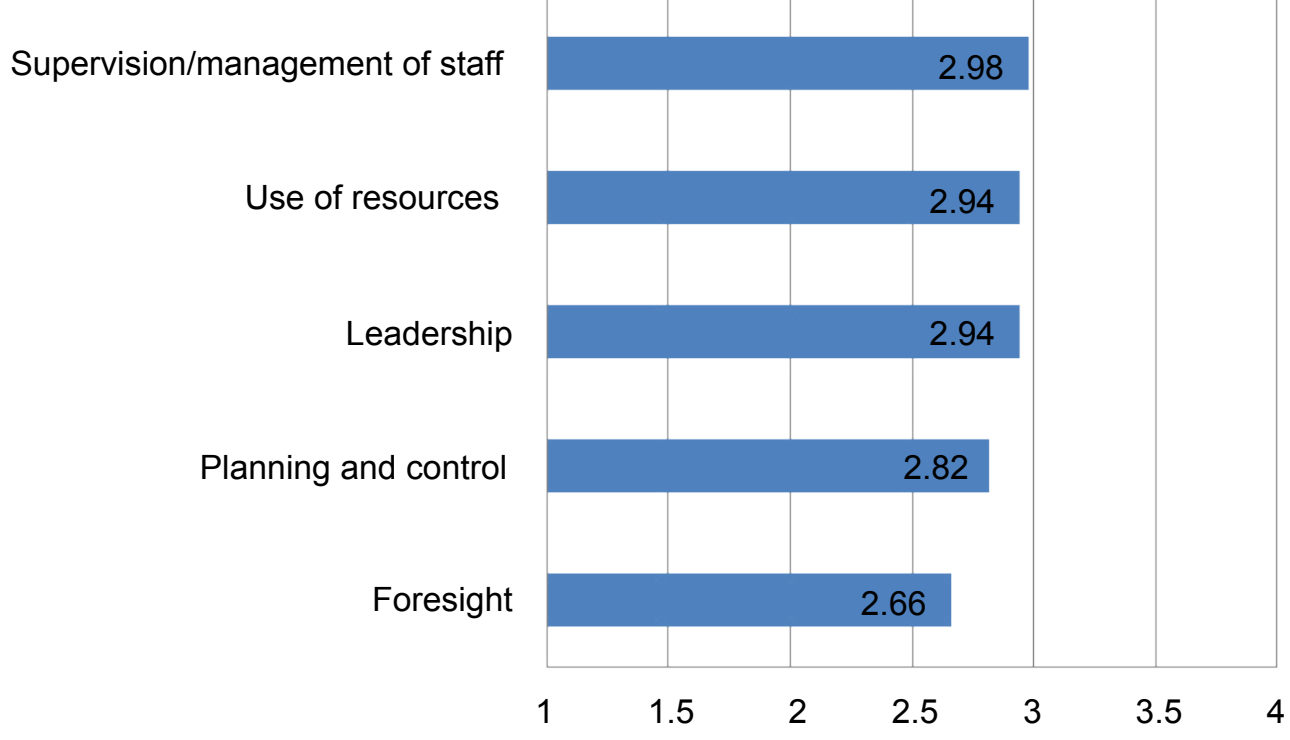

Figure 4: Mean of performance under managerial performance characteristics.

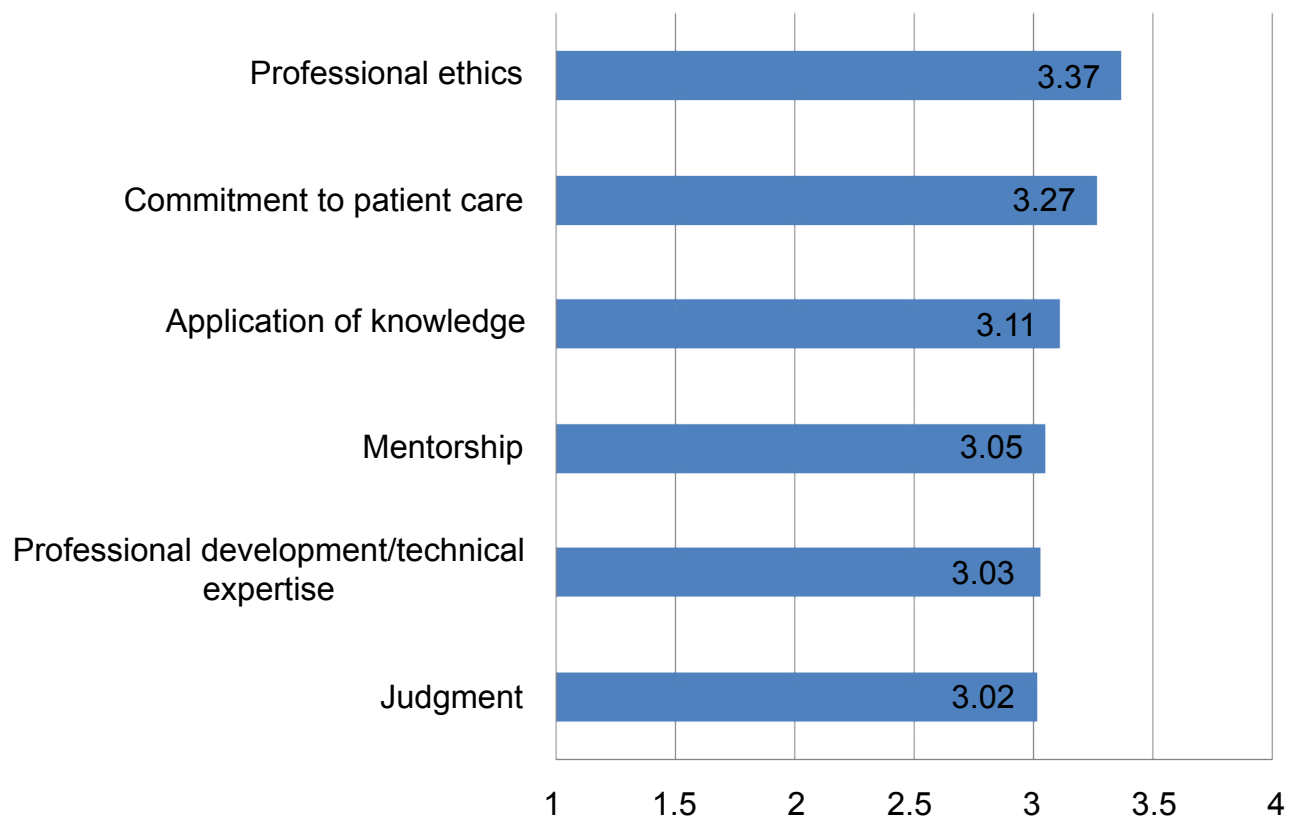

Figure 5: Mean of performance under professional performance characteristics.

influencer training, complaint management, motivational interviewing, competency-based interviewing skills, and decision making and analytical skills (advanced level) under general performance characteristics when comparing to the average mean of self-perceived training needs. Training needs related to advanced negotiate strategies, finance for executives, strategic talent management, strategic human resources management, power, politics \& empowerment, and leading \& managing change under managerial performance characteristics were necessary to be provided. Professional portfolio is another training need to be strengthened (Figure 6, Figure 7 and Figure 8).

\section{Training needs perceived by DOM/NC/SNO on WM}

The overall percentage of training needs from the perspectives of DOM/NC/SNO on WM was $73.37 \%$. WMs should receive the trainings of complaint management, influencer training, decision making and analytical skills (advanced level), constructive collaboration, developing professional assertiveness and confidence, team alignment, advanced writing, motivational interviewing, creating and leading a culture of innovation, gaps bridging, and competency-based interviewing skills under general performance characteristics when comparing the overall mean of percentage of training needs from their perspectives. WM needed to receive the trainings related to employee engagement, mediation and conflict management, leading \& managing change, performance management, leadership greatness, and authentic \& transformational leadership under managerial performance 
Media management Creating and leading a culture of innovation

Overseas Study visit Presentation skills to public/patients Influencer training

Complaint management Motivational interviewing Competency-based interviewing skills Decision making and analytical skill (advanced... Gaps bridging Team alignment Quality tools

Developing professional assertiveness and... Advanced evidence-based practice Constructive collaboration Project Management Advanced writing Stress management

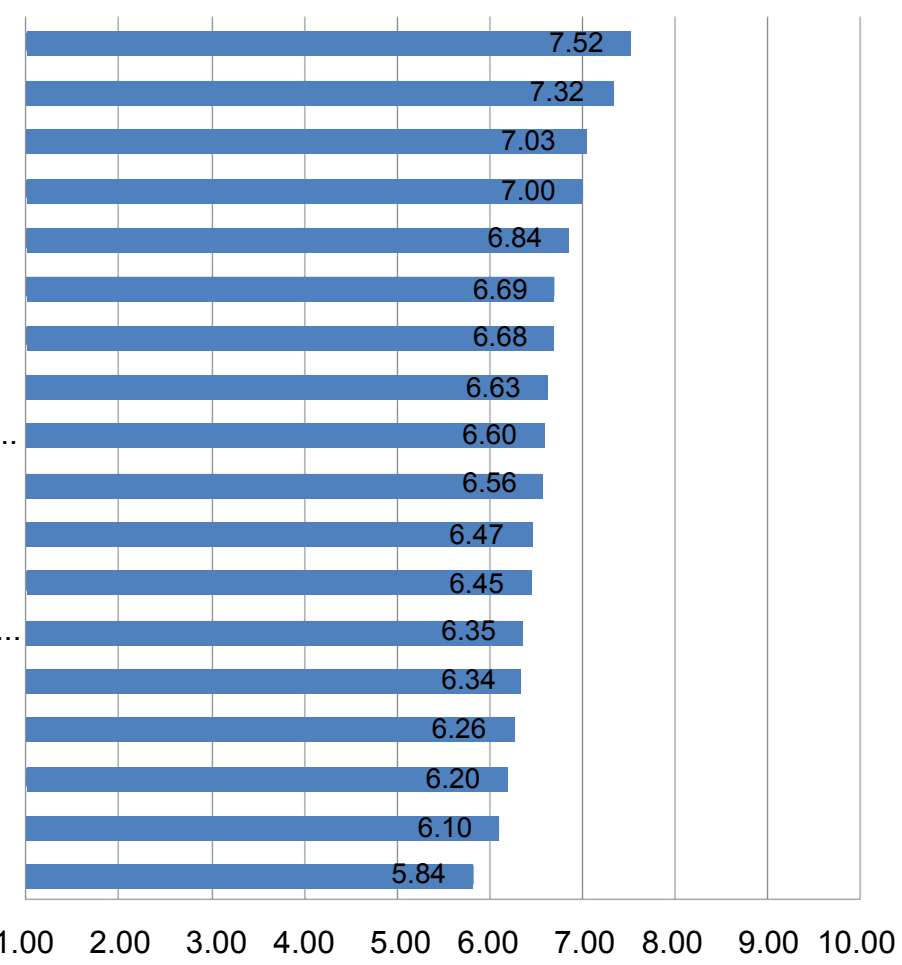

Figure 6: The mean of self-perceived training needs under general performance characteristics.

Advancced Negotiation strategies Finance for executives Strategic talent management

Strategic human resources management

Power, Politics \& empowerment Leading \& managing change

Authentic \& transformational leadership Employee engagement

Mediation and conflict management Leadership greatness Building social capital Performance management Motivation and empowerment Clinical leadership \& management skills...

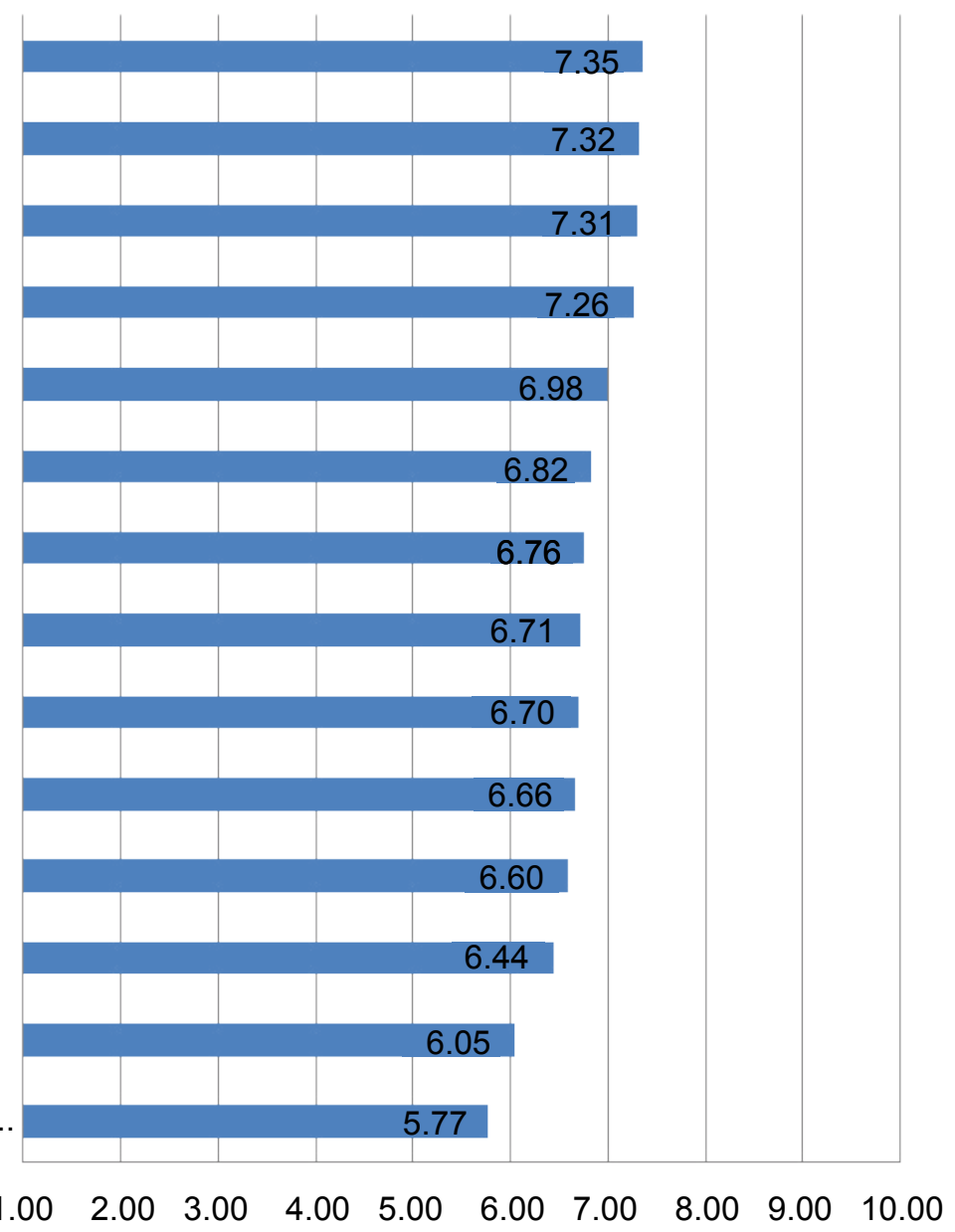

Figure 7: The mean of self-perceived training needs under managerial performance characteristics. 


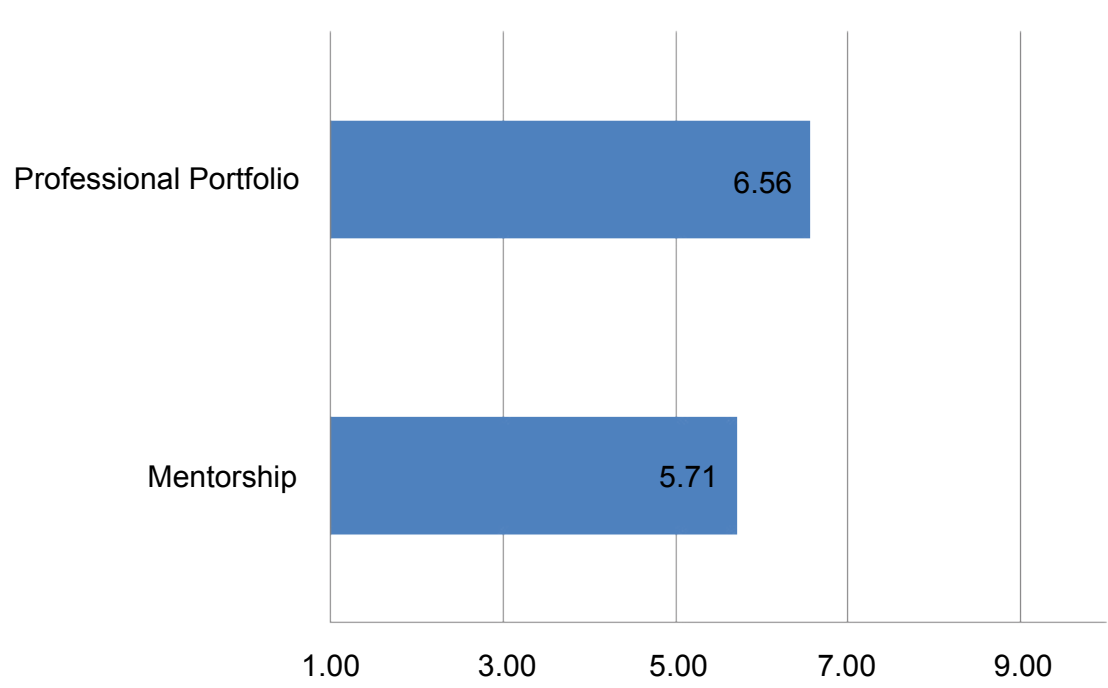

Figure 8: The mean of self-perceived training needs under professional performance characteristics.

\section{TN(DOM->WM) - General Performance Characteristics}

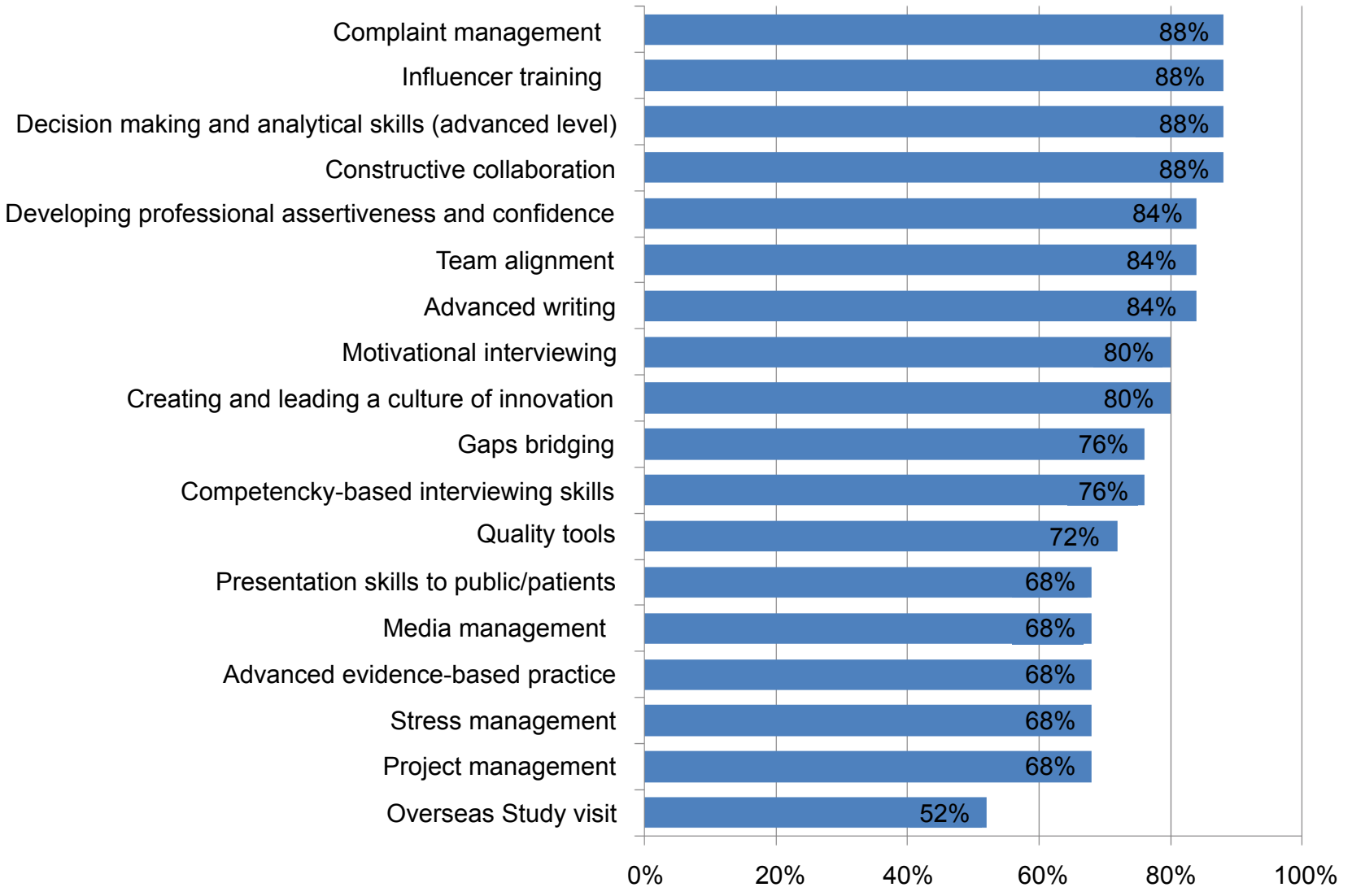

Figure 9: The mean of percentage of training needs from the perspectives of senior nurse managers on ward managers under general performance characteristics.

characteristics. They also needed to learn how to prepare their professional portfolios and to become a good mentor under professional performance characteristics (Figure 9, Figure 10 and Figure 11).

\section{Comparison of significant difference of self-perceived performance by years in rank}

Senior managers with less than 6 years of experience were prone to be insufficient in performing supervision and management of staff $(t=2.61, d f=60, \rho=0.011)$ (Figure 12).

Comparison of significant difference of self-perceived training needs by number of year in their present unit

Senior managers who had worked in their present 


\section{TN(DOM->WM) - Managerial Performance Characteristics}

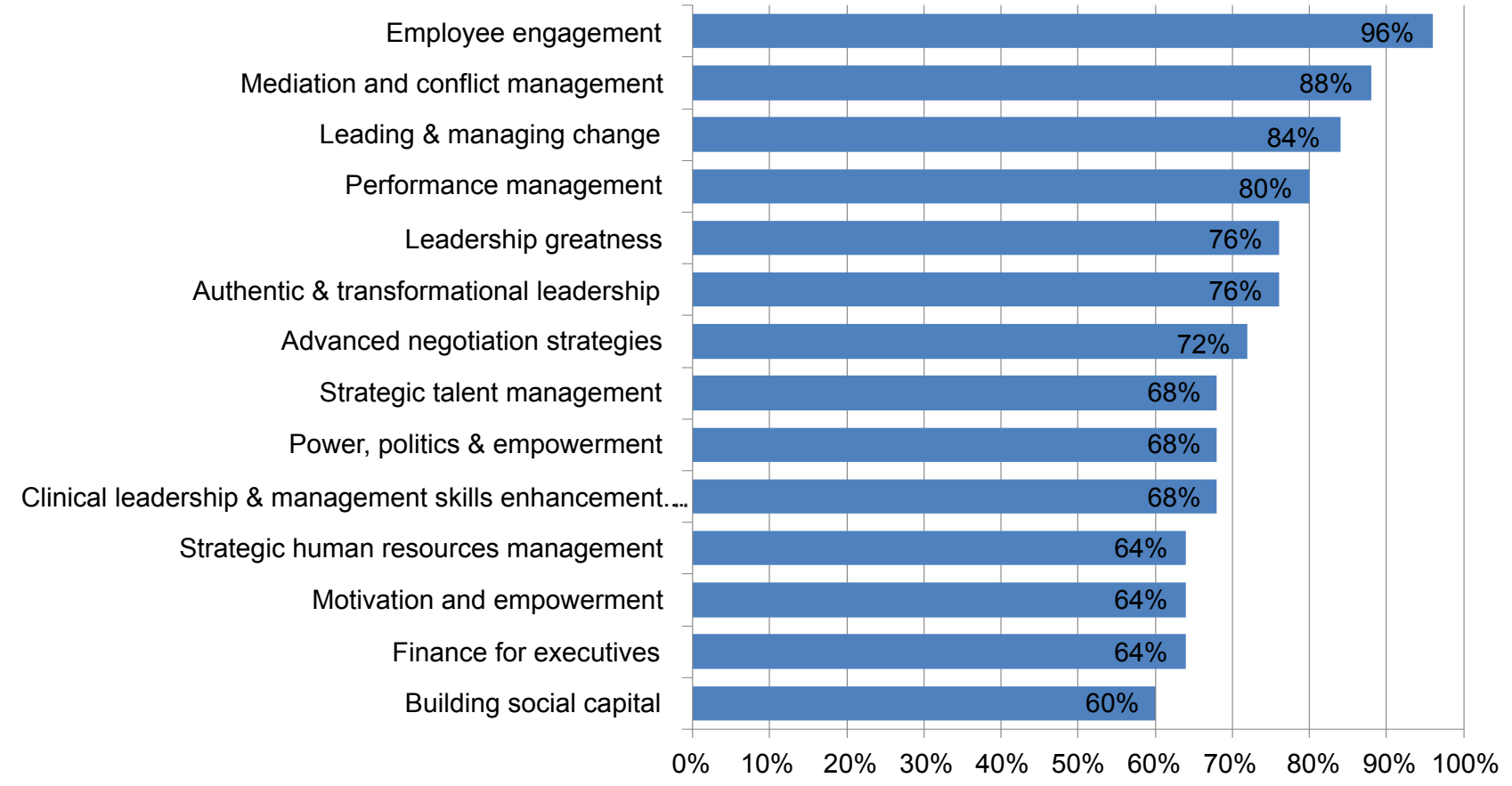

Figure 10: The mean of percentage of training needs from the perspectives of senior nurse managers on ward managers under managerial performance characteristics.

\section{TN(DOM->WM) - Professional Performance Characteristics}

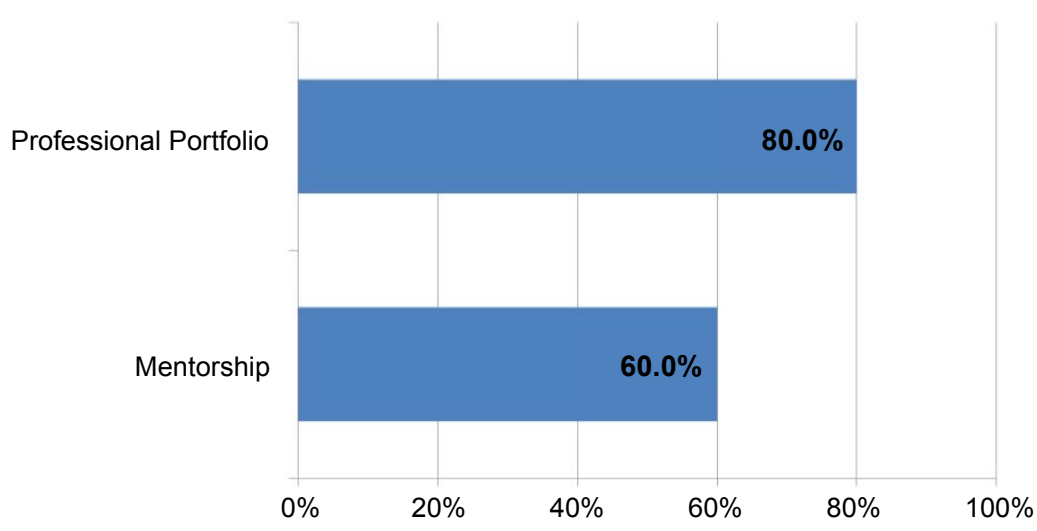

Figure 11: The mean of percentage of training needs from the perspectives of senior nurse managers on ward managers under professional performance characteristics.

unit for less than 6 years were necessary to receive more trainings on project management $(\mathrm{t}=2.68, \mathrm{df}=$ $59, \rho=0.01)$, quality tools $(t=2.32, d f=60, \rho=0.02)$, authentic $\&$ transformational leadership $(\mathrm{t}=2.27, \mathrm{df}=$ $60, \rho=0.027)$, leading \& managing change $(t=2.14, d f$ $=60, \rho=0.037)$, leadership greatness $(t=2.22, d f=60$, $\rho=0.031$ ), and clinical leadership \& management skills enhancement (Advanced Nurses Education workshop) $(t=2.27, d f=60, \rho=0.027$ ) (Figure 13a and Figure 13b).

\section{Comparison of significant difference of self-perceived training needs by years in their current rank}

Senior managers who had worked in their current rank for less than 6 year required more training on cre- ating and leading a culture of innovation $(\mathrm{t}=2.10, \mathrm{df}=$ $60, \rho=0.04$ ) (Figure 14).

\section{Professional development and training framework on performance characteristics for senior level managers}

A professional development and training framework on performance for senior level managers was developed according to the overall mean of self-perceived training needs (Table 2).

\section{Discussion}

A high response rate (84.9\%) of this study revealed that senior level managers were eager to reflect their self-perceived performance characteristics and train- 


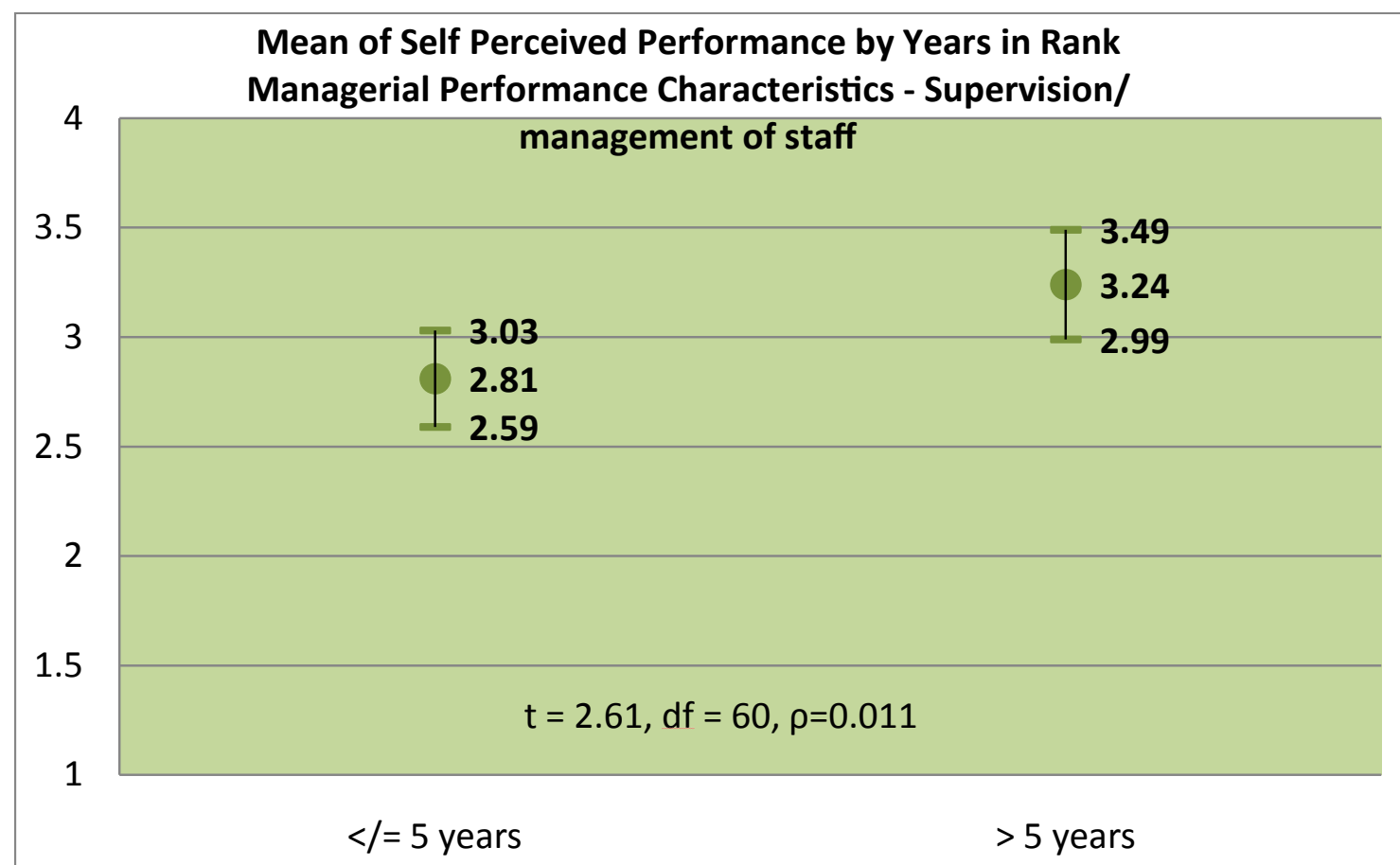

Figure 12: Comparison of significant difference of self-perceived performance by years in rank - managerial performance characteristics.
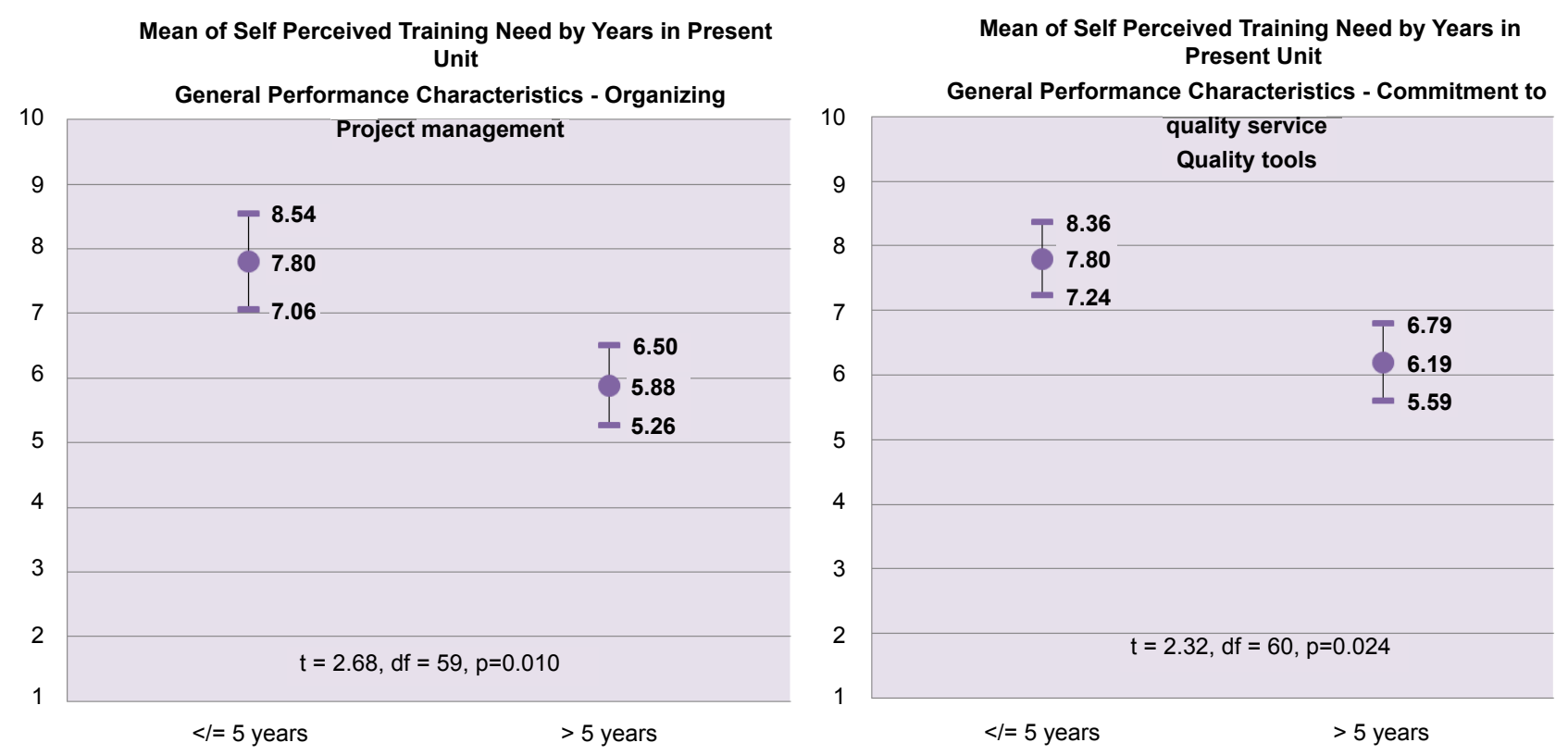

Figure 13a: Comparison of significant difference of self-perceived training needs by number of year in their present unit general performance characteristics.

ing needs. The main reason is that there was no similar study conducted in the past to explore the genuine training needs for senior level manager. They had demonstrated an open-minded attitude to express their opinion and increased recognition of the study that influenced their professional development and service development. In addition, it may be partly related to the strategy that senior level managers were given a plenty of time to complete the questionnaire during regular senior management meeting. It also reflected that senior level managers intended to apprehend the actual per- formance and training needs of themselves and others so that a succession plan can be developed based on the study.

Senior level managers with less than six years of experience in $\mathrm{UCH}$ accounted for 59.7 percent of this staff group. It implied that a large training opportunity is present to train them up as an effective leader in future. In this study, most of them needed training in the areas of leadership, supervision, staff and resource management, planning, and innovation that were underscored enough. It reflected that although they have 

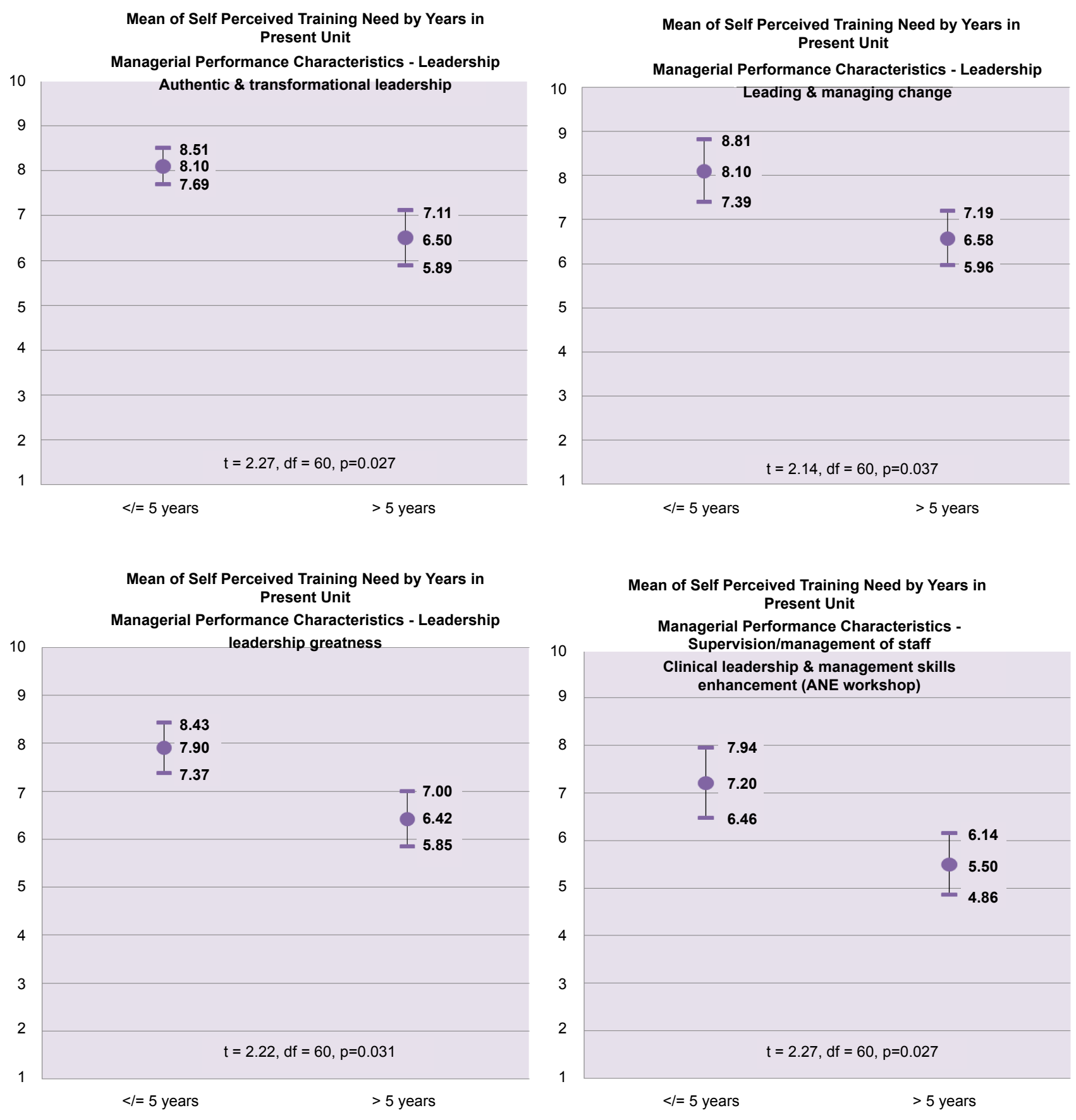

Figure 13b: Comparison of significant difference of self-perceived training needs by number of year in their present unit managerial performance characteristics.

been in a senior management position, the inadequate performance characteristics have been implied by comparing to the group of senior level managers over five years of experience that the younger senior level managers perceived lower mean of managerial performance characteristics (1.77) and higher mean of managerial training needs (6.97). These characteristics are essential in sustaining supportive workplaces and building the capacity and resilience of nursing workforces [23]. Moreover, a competent senior level manager shall strive to perform effective professional and high-quality care for other clinical managers and frontline nurses [24]. As a result, these trainings are necessary to be emphasized for young senior level managers.
There were few studies which examined the training needs of senior level manager and only a closely relevant study was identified. The design of the study adopted initial interviews with clinical nurse managers to provide in-depth, qualitative data [25]. However, the study was prone to focus on continuous professional development and job satisfaction enhancement that are self-centered. It requires nurses at different levels to improve the educational status of health professionals in order to meet these expectations [26]. Therefore, a variety of patient services led to many opportunities for professional development and experience exposure in different specialties. In other words, training programmes are also required to accommodate service development to meet patients' needs. 
Table 2: A professional development and training framework on performance for senior level managers.

\begin{tabular}{|c|c|}
\hline Performance sub-characteristics & Training Needs (descending order starting from the most needs) \\
\hline Relations with public/patients & Media management \\
\hline Planning and control & Advanced Negotiation strategies \\
\hline Innovation & Creating and leading a culture of innovation \\
\hline Use of resources & Finance for executives \\
\hline Leadership & Strategic talent management \\
\hline Use of resources & Strategic human resources management \\
\hline Innovation & Overseas Study visit \\
\hline Relations with public/patients & Presentation skills to public/patients \\
\hline Leadership & Power, politics \& empowerment \\
\hline Communication (verbal and written) & Influencer training \\
\hline Leadership & Leading \& managing change \\
\hline Leadership & Authentic \& transformational leadership \\
\hline Supervision/management of staff & Employee engagement \\
\hline Supervision/management of staff & Mediation and conflict management \\
\hline Relations with public/patients & Complaint management \\
\hline Communication (verbal and written) & Motivational interviewing \\
\hline Leadership & Leadership greatness \\
\hline Communication (verbal and written) & Competency-based interviewing skills \\
\hline Problem solving and decision making & Decision making and analytical skills (advanced level) \\
\hline Leadership & Building social capital \\
\hline Teamwork & Gaps bridging \\
\hline Professional development/technical expertise & Professional portfolio \\
\hline Teamwork & Team alignment \\
\hline Commitment to quality service & Quality tools \\
\hline Supervision/management of staff & Performance management \\
\hline Relations with public/patients & Developing professional assertiveness and confidence \\
\hline Commitment to quality service & Advanced evidence-based practice \\
\hline Organizing & Constructive collaboration \\
\hline Organizing & Project management \\
\hline Communication (verbal and written) & Advanced writing \\
\hline Supervision/management of staff & Motivation and empowerment \\
\hline Problem solving and decision making & Stress management \\
\hline Supervision/management of staff & Clinical leadership \& management skills enhancement (ANE workshop) \\
\hline Mentorship & Mentorship \\
\hline
\end{tabular}

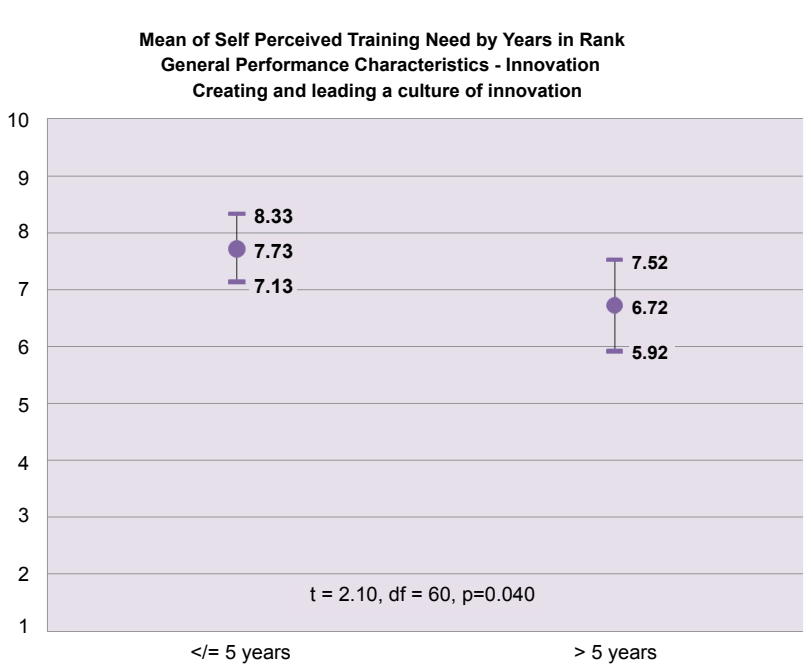

Figure 14: Comparison of significant difference of selfperceived training needs by years in their current rank general performance characteristics.

A nursing professional development specialist has, undoubtedly, a role on successful succession planning, managing competing priorities, and effecting cost avoidance [27]. Having a leadership role, we intend to conduct this research study to explore continuing education program development, redesign the existing training systems, and plan training strategically. Through the study, senior level managers examine their learning needs and their career development in their specialties. The effective functioning of the healthcare system, on the other hand, is highly dependent on a competent health services management workforce [28]. We indeed require an effective tool to study the financial implications of workforce and dynamic changes of manpower. However, the effectiveness of the current healthcare management workforce is influenced by health reforms, the aging of one's country health workforce, and health systems [28]. These factors preclude the training planning unless we understand the real training needs from staff's perspectives. This study has guided the nursing professional development specialists to enhance their competences and performance characteristics for respective trainings for senior level managers so as to prevent mis-allocation of resources. We address their needs to provide related training accordingly; for example, media management training that ranked the highest need has not been held before and we may invite 
appropriate speaker to deliver the training for senior managers. Thus, it can better support senior level managers in achieving their role within healthcare systems [29].

A key strength of the study was to analyse self-perceived performance characteristics and training needs towards different domains including personal growth, service development and professional development. Based on the discussion of the focus group, the three domains identified are the core elements to guide nurses on how to achieve the performance characteristics. In the past, NSD provided few training sessions related to personal growth which describes people's change and development throughout the life span [15]. Personal growth is highly related to role modelling and being used as a resource [30]. Through the study, we understand what kind of trainings should be given in order to balance their work life. Respondents may also learn what they should change through taking part in the TNA periodically which acts as transactional analysis and a systematic psychotherapy for personal growth and personal change [31].

In future, the training needs analysis survey should be conducted periodically so as to monitor the dynamic changes of training needs perceived by senior level managers. Staff attrition, promotion, and recruitment are absolutely influential in the actual training needs. In addition, the analysis of training needs especially in evaluation should not stop when the framework is structured and implemented [32]. A full scope of evaluation is employed to facilitate on-going analysis. Educator needs to measure outcomes that provide data for future TNAs.

\section{Implications}

A middle level manager requires actual training needs based on self-perceived performance characteristics but does not require a top-down training plan. This study identified a variety of trainings that could improve the process of general, managerial, and professional development thus supporting DOM/NC/SNO and WM in achieving their role within health-care systems in $\mathrm{UCH}$. Moreover, it is an imperative to identify a gap of what a leader should require to develop a succession plan at different levels.

\section{Limitations}

Although this study had identified essential training needs for senior level managers, they need to be interpreted in light of some limitation in this study. Many training programmes have been listed in the questionnaire but some are not included. Nevertheless, respondents might write down other training needs they intend to have.

On the other hand, there are different ways to collect relevant information from nursing staff. Norman, et al. (2004) [33] illustrated some examples, such as practice experience, reflection, questioning, practice audits, self-assessment tests, peer interview and other sources. As it was considered that a structural training framework should be designed in the upcoming years, a survey is the most appropriate TNA data collection tool. Some argued that a questionnaire including lists of training needs as options might shape the respondents to consider the itemized training needs solely and neglect other potential trainings. This issue was brought to discuss in our study panel and came to a conclusion that such questionnaire design could stimulate and guide the respondents to perceive their training needs based on the three major domains despite the preconceived bias and notions of the research team probably.

The rating of training needs on subordinates was limited to using a dichotomous response format because the number of WMs was relatively lower compared to other ranks. Another limitation of the study is its small sample size of senior nurse manager, which was focusing on one public hospital in Hong Kong only, recruited through convenience sampling.

\section{Conclusions}

The findings of this study suggest that senior nurse managers need relevant education on relations with public/patients, planning and control, innovation, leadership, use of resources, communication (verbal and written), and supervision/management of staff to enhance professional nursing practice and support achievement of career goals. Evidently, senior nurse managers with less than 6 years of experience require more education on some specific areas. In light of this study, the nursing professional development specialist can develop the framework for education and professional development to meet senior level managers' needs in the upcoming 3 years.

\section{Acknowledgement}

The authors would like to express their sincere gratitude to all DOMs, NCs, SNOs and WMs for participating in this study.

\section{Funding}

This research received no specific grant from any funding agency in the public, commercial, or not-forprofit sectors.

\section{Conflict of Interest}

No conflict of interest has been declared by the authors.

\section{References}

1. Devi VR, Rao MM (2012) Training needs identification of nursing staff - a case study of a health care organization. EXCEL International Journal of Multidisciplinary Management Studies 2: 147-153.

2. Brown J (2002) Training needs assessment: A must for developing an effective training program. Public Personnel Management 31: 569. 
3. Salas E, Cannon-Bowers JA (2001) The Science of Training: A Decade of Progress. Annu Rev Psychol 52: 471-499.

4. Hamilton S (2013) Assessing knowledge skills in the NHS: a training needs analysis approach. Health Info Libr J 30: 168-173.

5. Gould D, Kelly D, White I, Chidgey J (2004) Training needs analysis. A literature review and reappraisal. Int J Nurs Stud 4: 471-486.

6. Dierdoff CE, Surface EA (2008) Assessing Training Needs: Do Work Experience and Capability Matter? Human Performance 21: 28-48.

7. Hospital Authority (2005) Core Competency for Enrolled Nurses, Registered Nurses \& Advanced Practice Nurses in Hospital Authority. Revised in October 2005 COC (N) Paper 13/2005.

8. Denscombe M (2007) The Good Research Guide for Small Scale Social Research Projects. (3 ${ }^{\text {rd }}$ edn), McGraw-Hill/ Open University Press, Maidenhead, Berkshire, UK.

9. Happell B (2007) Focus groups in nursing research: an appropriate method or the latest fad? Nurse Res 14: 18-24.

10. Lannon S (2007) Leadership skills beyond the bedside: professional development classes for the staff nurse. J Contin Educ Nurs 38: 17-23.

11. Gould D, Drey N, Berridge E (2007) Nurses' experience of continuing professional development. Nurse Educ Today 27: 602-609.

12. Ulrich B, Buerhaus $P$, Donelan K, Norman L, Dittus R (2005) How RNs view the work environment: Results of a national survey of registered nurses. J Nurs Adm 35: 389-396.

13. Cooper E (2009) Creating a culture of professional development: a milestone pathway tool for Registered Nurses. J Contin Educ Nurs 40: 501-508.

14. Sinha D, Sinha S (2009) Training needs analysis. Personal growth and training \& development. India: Word-press, 139-148.

15. Weigold IK, Weigold A, Russell EJ, Drakeford M (2014) Examination of the psychometric properties of the Personal Growth Initiative Scale-II in African American college students. Assessment 21: 754-764.

16. Robitschek C, Ashton MW, Spering CC, Geiger N, Byers D, et al. (2012) Development and psychometric evaluation of the Personal Growth Initiative Scale-II. J Couns Psychol 59: $274-289$.

17. IBM Corp. Released (2015). IBM SPSS Statistics for Windows Version 23.0. IBM Corp, Armonk, NY.
18. MaCorr Research (2014) Sample size calculation.

19. Yaghmaie $F$ (2003) Content validity and its estimation. Journal of Medical Education 3: 25-27.

20. Polit DF, Beck CT (2006) The content validity index: are you sure you know what's being reported? Critique and recommendations. Res Nurs Health 29: 489-497.

21. Nasrin P, Trisha D (2009) Developing and Validating a Questionnaire to Measure Spirituality: A Psychometric Process. Global Journal of Health Science 1: 2-11.

22. Streiner DL, Norman RG (2003) Health measurement scales: a practical guide to their development and use. $\left(5^{\text {th }}\right.$ edn), Oxford University Press.

23. Mannix J, Wilkes L, Daly J (2013) Attributes of clinical leadership in contemporary nursing: an integrative review. Contemp Nurse 45: 10-21.

24. Martin JS, Mccormack B, Fitzsimons D, Spirig R (2012) Evaluation of a clinical leadership programme for nurse leaders. Journal of Nursing Management 20: 72-80.

25. Gould D, Kelly D, Goldstone L, Maidwell A (2001) The changing training needs of clinical nurse managers: exploring issues for continuing professional development. J Adv Nurs 34: 7-17.

26. Yfantis A, Tiniakou I, Yfanti E (2010) Nurses' attitudes regarding Continuing Professional Development in a district hospital of Greece. Health Science Journal 4: 193-200.

27. Swihart D (2009) Nursing professional development: roles and accountabilities. Medscape.

28. Liang Z, Leggat SG, Howard PF, Koh L (2013) What makes a hospital manager competent at the middle and senior levels? Aust Health Rev 37: 566-573.

29. Pegram AM, Grainger M, Sigsworth J, While AE (2014) Strengthening the role of the ward manager: a review of the literature. Journal of Nursing Management 22: 685-696.

30. Cotterill-Walker SM (2012) Where is the evidence that master's level nursing education makes a difference to patient care? A literature review. Nurse Educ Today 32: 57-64.

31. Lall M, Sharma S (2009) Transactional Analysis. Personal growth and training \& development. ( $1^{\text {st }}$ edn), Excel Books, New Delhi, 134.

32. Pilcher J (2016) Learning Needs Assessment: Not Only for Continuing Education. J Nurses Prof Dev 32: 185-191.

33. Norman GR, Shannon SI, Marrin ML (2004) The need for needs assessment in continuing medical education. BMJ 328: 999-1001. 